\title{
Using in-car feedback to influence travel decisions
}

\author{
I. T. Byrne, M. M. O'Mahoney, B. M. Broderick and W. D. E. O'Sullivan
}

\section{P. O'Neill, Traffic Solutions Limited}

The authors have conducted an interesting piece of research which has demonstrated the potential for larger-scale behavioural projects of this type, and they have achieved intuitively plausible results despite the small sample available to them.

They observe that divergence between market prices and resource costs lead to misallocation of economic resources, but it could be pointed out that direct cost of travel to the individual motorist includes a large element of fuel taxation which is in part a proxy for the total costs to society additional to those embodied in the resource (non-taxed) cost of the fuel. Accordingly, it would be of interest to know what cost levels were notified to drivers in the 'external cost' element of the trials. Because this is composed largely of delay costs imposed on other road users, it is sensitive to current levels of traffic congestion; an attempt to reflect this was included in the innovative method of congestion pricing proposed for the city of Cambridge, England, but this required sophisticated monitoring equipment. The figures used in Dublin were presumably not dynamic; did they, however, embody the very different levels appropriate to peak journeys to work as opposed to off-peak leisure trips? Were the costs presented to drivers expressed in pence per mile, or as an aggregate cost for each trip? In the latter case, drivers would be able at once to compare this with the known cost of using public transport, and it might be expected to have a greater impact on their subsequent decisions on trip options and mode choice. The fuel costs and non-fuel marginal costs have evidently been deduced from evidence provided by the subjects themselves, but clearly they are not consistently accurate. Although these other mileage-dependent costs will vary with car size and model, they are likely generally to lie in the range 0.5-0.9 times fuel cost, and figures outside this range should receive careful scrutiny. Major elements (tyres, brakes, etc.) may require replacement intervals of two or three years and a long history is needed if their costs are to be correctly estimated. The use of a derived function for instantaneous fuel consumption appears to be based only on running speed (regardless of gear selected?), whereas actual consumption is highly dependent on engine loading (including gradient and acceleration), while driving style has an important effect on the costs attributable to brake and tyre wear. Was any account taken of these factors?

\section{Authors' reply}

The authors welcome the discussion contribution of Mr. 0'Neill and his interest in their research. They are also grateful to him for taking the time to comment.

The authors fully accept and recognise this point regarding fuel taxation. They agree that fuel taxation could act as a rough proxy for the total costs of car travel to society but given that fuel taxation is not currently hypothecated for transport investment, or certainly not in a way that is directly recognisable, theoretically this approach is not strictly correct. While the authors accept that some of the taxation will indirectly be invested in transport by default, they would argue that car drivers perceive fuel taxation only as a means of general revenue generation by a government and not as a means of offsetting the external costs of travel. The authors would argue that imposing a charge on a car driver that is clearly identified to them as a means of offsetting the external costs of travel could quite possibly have a different effect than if the charge, such as current fuel taxation, is seen as for general revenue generation. This point was indirectly proven in the research where few of the individuals recognised the high costs their car travel were imposing on society.

The external cost included delay costs in a dynamic way. This was done by applying a higher rate of charge for the time during which the vehicle was travelling less than $20 \mathrm{~km} / \mathrm{h}$, representing delay in congested situations. The rates used were derived from the result of the TRENEN model (EU DGVII funded research) which estimated the external costs of travel, including congestion, delay and accidents. Specific rates were not applied for peak and off-peak period travel but the higher rates for travel in queuing conditions, as mentioned above, were sufficient to ensure that the aggregate cost of a trip in the peak period would be considerably higher than a similar trip in the off-peak period.

The costs were presented to the driver in two ways: pence per mile and as an aggregate cost for each trip. An objective measure of fuel was conducted by filling the tank with fuel, driving the vehicle in typical urban conditions, noting the distance and refilling the tank. There was no option to use fuel meters in this research given the dangers involved. Under the circumstances the authors consider that the 
method they used was sufficient for the purposes of the work.

The authors take the point that non-fuel marginal costs are difficult to estimate but in theory the whole life history of the vehicle needs to be examined and this was not possible. The authors used a one-year history because the car drivers could not recall their maintenance expenditure accurately two or three years before. A subtle but very important point that requires noting here is that while it is important to estimate and present costs accurately, an equally important point is ensuring that the drivers believe the costs presented to them.
While this may seem to be too subtle to be relevant, the authors found that it was a very important point in this research.

The authors are well aware of the factors influencing fuel consumption. As mentioned previously, the use of fuel meters was not an option given the dangers involved. All car meters were calibrated for fuel consumption using a standard urban driving cycle and the authors consider this to be sufficient for the purposes of the work. Driving style was addressed in one phase of the research concentrating on long journeys where car drivers were presented with the impact on cost by varying their speed. 